\title{
A Low Cost, High-Reliability Receiver System for Reading Passive RFID Tags
}

\author{
Nicollas R. de Oliveira $^{1}$ (D), Lucas P. Boaventura ${ }^{2}$ D, Tadeu N. Ferreira ${ }^{2}$ (D) \\ Vanessa P. R. Magri ${ }^{2}$ (D) Jacqueline S. Pereira ${ }^{2}$ (D) Diogo M. F. Mattos ${ }^{1}$ (it) \\ ${ }^{1}$ LabGen/MídiaCom - PPGEET/TET/UFF, Universidade Federal Fluminense, Niterói, RJ, Brazil, \\ nicollas_rodrigues@id.uff.br,menezes@midiacom.uff.br \\ ${ }^{2}$ LAPROP - PPGEET/TET/UFF, Universidade Federal Fluminense, Niterói, RJ, Brazil, lucaspb@id.uff.br, \\ tadeu_ferreira@id.uff.br,vanesssamagri@id.uff.br, jacnovo@gmail.com
}

\begin{abstract}
Radio-Frequency Identification (RFID) is one of the leading enabling technologies of the Internet of Things (IoT) at low cost and high granularity scenarios. The cost of implementing IoT applications concerns the development and operation of receiver systems for reading labels. In this paper, we propose a low-cost receiver system based on off-the-shelf components allied to the development of a microstrip antenna. The proposed system is capable of receiving and processing digital signals scattered back from a passive RFID tag. The proposal relies on a Python application embedded in Raspberry $\mathbf{P i}$ hardware, in which a bank of digital filters processes the signal. The proposal separates the received signal into sub-bands and implements a decision-maker that detects and codes radiofrequency carriers' presence in sub-bands. Simulation using artificial random noise proves that the proposed and implemented decision-maker achieves $92 \%$ reliability for a Signal-to-Noise ratio between $1.6 \mathrm{~dB}$ and $2.3 \mathrm{~dB}$. The evaluation of the printed dipole antennas pair for the RFID receiver system indicates a return loss of $19.22 \mathrm{~dB}$ for operation at $915 \mathrm{MHz}$.
\end{abstract}

Index Terms - Filter bank, Internet-of-Things, Microstrip antenna, RFID reader

\section{INTRODUCTION}

Radio-Frequency IDentification (RFID) is a widespread technology that identifies a particular object or user based on its characteristic radiofrequency (RF) [1] parameters. Its operation relies on the interaction of electromagnetic waves between a tag and a reader in the same RF range. The tag is a device that identifies the object to which it attaches, while the reader is the device that interfaces with external data processing systems [2]. Besides, RFID popularity led to the development of standards, such as ISO11784, ISO11785 [3], and the ANATEL (Telecommunications National Agency of Brazil) Resolution 680 [4]. Consequently, with the spread of use and the drop in tags' costs, RFID technology is identified as one of the leading enabling technologies for intelligent applications in the Internet of Things (IoT) [5], [6]. Identifying objects, people, and products makes the technology a perfect fit for unusual applications, such as assisting in the mobility of the elderly [7], the detection of moisture in disposable diapers [8] and the identification of animals [9].

Three key factors still limit the use of RFID technologies: (i) energy savings, which is mandatory to consider active tags that depend on power from batteries or external circuits; (ii) scale manufacturing costs, which is a recurring factor in conventional RFID systems that use integrated circuits or microchips; (iii) efficiency of transmission and reception. The scope of this paper focuses on reception, as there 
are strict requirements in noisy environments for the selectivity of filters, which are fundamental for separating combined signals [10], [11].

In this paper ${ }^{1}$, we propose a receiver system built using an application embedded in low-cost hardware, such as a Raspberry $\mathrm{Pi}$, and we also propose a pair of dipole microstrip antennas, operable at $915 \mathrm{MHz}$, to transmit and receive RFID signals. The emulation is developed in Python, using the digital filter bank technique to separate the received signal into sub-bands, detect and encode the presence and the absence of radio-frequency carriers. The pair of dipole antennas is evaluated in the laboratory, comparing simulated and experimental results.

Previous work describes millimeter-sized identification chips operating in terahertz frequencies, with relatively low-energy levels supplied by photovoltaic diodes [13], proposes chipless tags operating in gigahertz frequencies [11], or develops a UHF tag reader [14]. Besides, other works deploy experimental antennas for RFID receivers [15], [16]. Unlike previous works, we focus on developing a realistic simulation of a filter bank for separating chipless-tag signature frequencies and also on proposing a dipole antenna prototype that achieves a reflection coefficient of $-19.22 \mathrm{~dB}$ for the Brazilian standardized range of RFID operation. It is noteworthy that the proposed RFID system improves the performance of a professional RFID reader with low-cost components.

The remaining of the paper is organized as follows. Section II describes a brief theoretical background on the RFID system. Section III exposes the proposed system, including the modules that make up its architecture. In Section IV, the stages of developing the baseband emulation are detailed. Our results are highlighted in Section V. In Section VI, we present a practical approach to the design and manufacture of a receiver antenna suitable for RFID readers. Section VII reviews the related work. Finally, Section VIII concludes the paper.

\section{RADIO-FREQUENCY IDENTIFICATION OPERATION}

RFID operation resides on the communication between the reader and tags, as seen in Figure 1. Tags are electronic labels capable of storing a unique identifier called electronic product code (EPC). A reader wirelessly accesses the stored data upon a request signal. The general scheme of the proposed system is shown in Figure 1. Baseband processing, DAC and ADC are addressed in previous work [17] and [14]. Di Renna et al. explore reader antenna designs [18], tag designs, and evaluations [11].

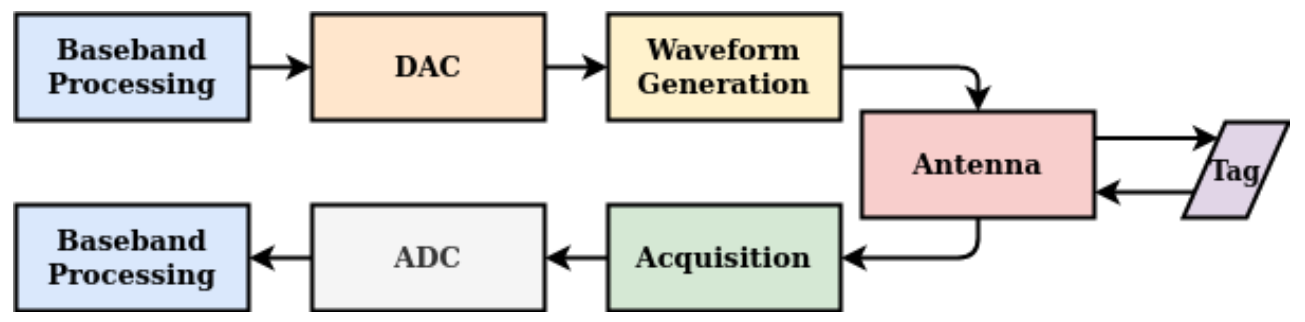

Fig. 1. The general scheme of our whole RFID project.

Active tags require a continuous power supply from a battery to work correctly. These tags usually encompass three main components: an on-board micro-controller, a memory, and a receiver. A power source enables the tags to initiate communication without waiting for a request and improves their reading range by hundreds of meters. Active tags, however, imply a greater weight, size, energy, and

\footnotetext{
${ }^{1}$ The paper is an extension of a previous conference paper [12] (in Portuguese).
} 
maintenance cost. Differently, passive tags harvest the power for their operation from the reader's emitted signal. The tag stores energy in a capacitor, and the communication usually relies on backscattering [19]. Since they are independent of a power source, passive tags show almost unlimited life, small size, little weight, and low cost. Such scarcity of available energy directly impacts the communication range, limited to a few meters. Semi-active tags combine features from both sorts of tags. A battery supply assists semi-active tags, but tags also absorb the power necessary for communication from the reader's magnetic field [20]-[22].

Readers comprise signal generation, transmission, reception, and processing. The reading procedure begins with generating a request signal. In this paper, the reading procedure consists of one or more sinusoidal signals at different frequencies. Thus, the request signal passes through a modulator that guarantees translation to an RF range of frequencies. Subsequently, the request signal goes through a power amplifier, ensuring that the destination receives the transmitting antenna's information at acceptable intensity levels. Once the request signal reaches the passive tag, it is scattered in several directions, eventually reaching the reader back. After the receiving antenna captures the signal, the receiver catches the signal through a symmetrical system to the transmission, passing it through the amplifier, demodulator, and finally processing it [23].

\section{THE PROPOSED RECEIVER SYSTEM}

Our proposal is organized into two stages, one emulates the reception of an RFID system, developed in Python language, and the other aims to design and manufacture a pair of dipole antennas operable at $915 \mathrm{MHz}$ for RFID readers. The proposed system's architecture, expressed in Figure 2, is modular and forecasts the complementary operation of two elements, a transmitter, and a receiver/processor. Initially, the Signal Generation module constructs and samples the request signal that one or more RF carriers may form. The Noise Addition module inserts noise components into the request signal to emulate a more realistic scenario. The noise emulates transmission over a real radio channel. Then, the Transmission module wirelessly sends the signal formed at the transmitter via a network socket. Once connected to the same local network as the transmitter, the receiver/processor picks up the request signal via the Reception module. Subsequently, the Filter Bank module [24] associates digital filters to separate the received signal into sub-bands. The Decision-maker (detection) module calculates the average signal and noise powers in the spectral domain and compares them to establish a coding procedure, translating the existence and the absence of carriers in a binary identification code. Finally, the Sensitivity Analysis module allows evaluating the decision maker's performance in different ranges of signal-to-noise ratio (SNR).

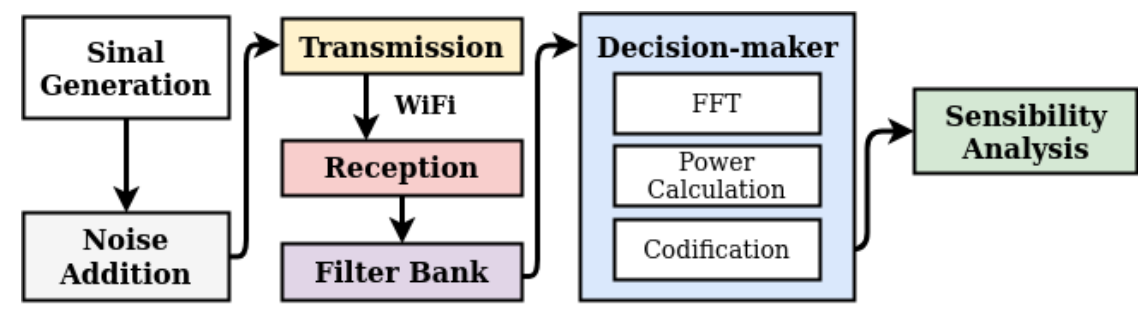

Fig. 2. Emulation of the RFID transceiver system, which implements modules for signal transmission and reception for validation. A WiFi network emulates the aerial interface, included emulated noise.

The setup is intended to communicate with the tags fabricated in a previous stage of this project. Di 
Renna et al. describe the tags [11] depicted in Figure 3. Mota et al. address the transmitted waveforms in Labview [17].

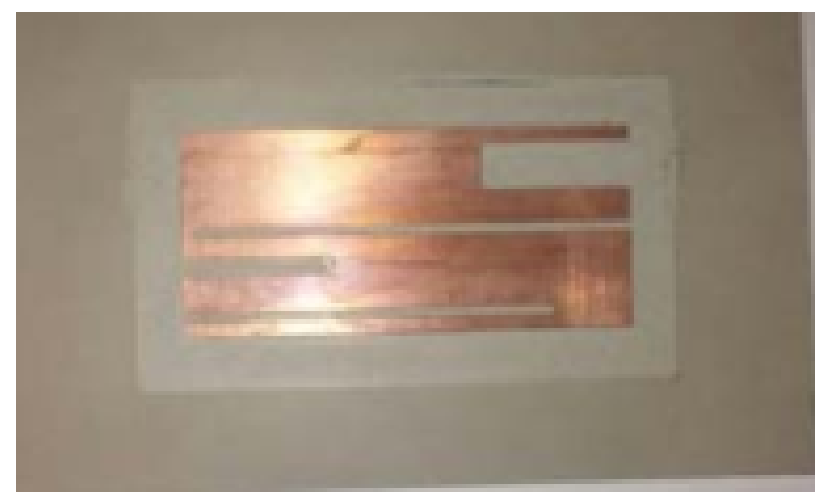

Fig. 3. Tags fabricated in a previous stage of this project [11].

\section{EMULATION SCENARIO}

The proposed emulation essentially consists of the interaction between two elements: a transmitter and a receiver, represented by a notebook and a Raspberry Pi. It is worth mentioning that the emulation runs on a personal notebook equipped with an Intel Core i7 4770 processor, with 8 GB of RAM and 1 TB of hard disk, and a Raspberry Pi 3B microprocessor.

\section{A. Transmission of Request Signals and Random Noise}

The emulation procedure begins at the transmitter that produces the request signal, composed of a pair of cosine carriers at $42 \mathrm{MHz}$ and $84 \mathrm{MHz}$, sampled at $200 \mathrm{MHz}$. Adopting these frequency values aims to align the proposed system with the values presented in the transmission stage in previous work [14].

We generate a uniform random noise to emulate the effects of noise in a real transmission scenario. We deploy scipy library [25] available for the Python language to generate the noise, which we artificially add to the request signal. Although the library functions allow the choice of the number of random samples that make up the noise, the signal-to-noise ratio (SNR) value is not controlled. Instead, it only allows configuration of the noise variation range bounded by its upper $L_{\text {sup }}$ and lower $L_{\text {inf }}$ limits. Being aware of this interference on the SNR, the alternative is to establish a relationship between the upper and lower limits of the noisy signal with the maximum values $x_{\max }$ and minimum $x_{\min }$ request signal through a variable entitled correction factor $\left(F_{c o r r}\right)$. The variables are related according to:

$$
\begin{gathered}
L_{\text {sup }}=x_{\text {max }} F_{\text {corr }} \\
L_{\text {inf }}=x_{\text {min }} F_{\text {corr }} .
\end{gathered}
$$

Once the signal is composed, it is transmitted from the notebook to the microprocessor through a local network that uses the IEEE 802.11 (WiFi) standard. Computationally, network sockets enable communication. We chose WiFi technology because it has a low cost and it is available on the Raspberry Pi [6]. After receiving the signal, the Raspberry Pi begins the processing measures described below. 


\section{B. FILTER BANK PROCESSING}

An analysis filter bank consists of an array of filters designed to separate a signal into sub-bands [26]. Each sub-band may be processed independently. Due to the selectivity and linear phase requirements, we design a bank of orthogonal digital FIR (Finite Impulse Response) filters composed of a low-pass orthogonal filter of order 50 and a high-pass of order 51 , using $50 \mathrm{MHz}$ as the cutoff frequency.

The filter bank's impulse response is convolved with the received signal, using the function convolve of the library numpy. Then, the phenomenon presented in Figure 4 is observed. As expected, the figure shows that the original request signal, composed of a pair of carriers, was adequately broken down into two filtered signals, one containing the carrier at $42 \mathrm{MHz}$ and the other at $84 \mathrm{MHz}$.
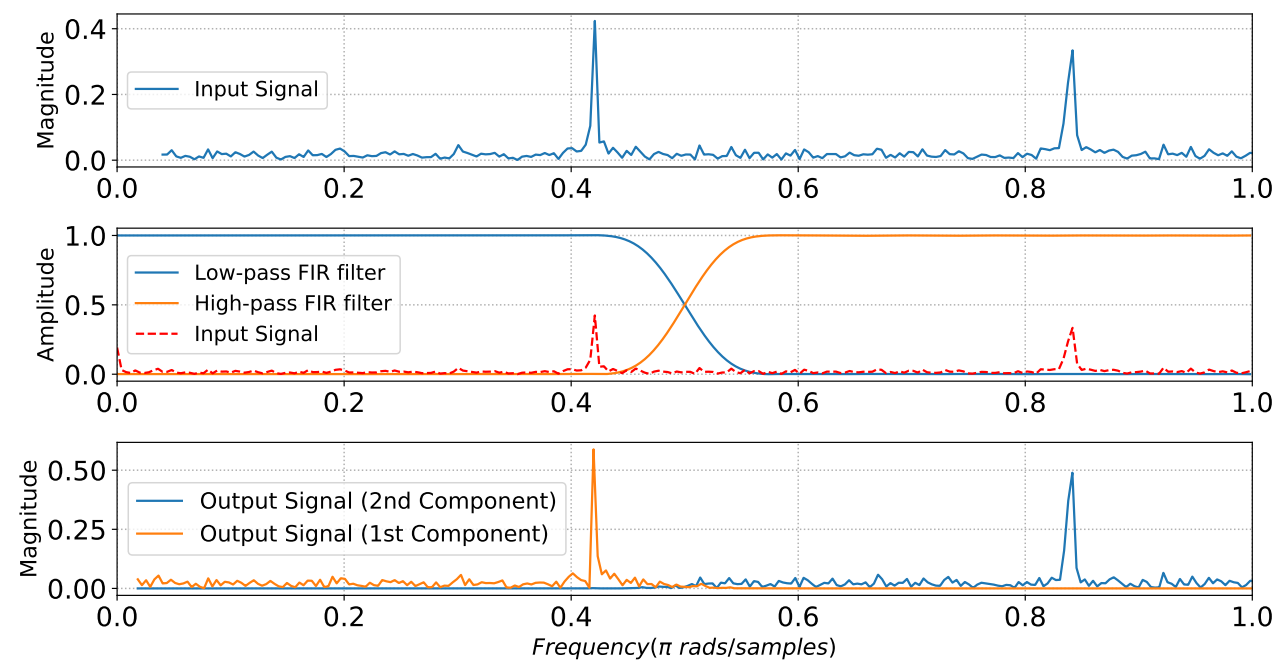

Fig. 4. Frequency-domain magnitude representation of: a) the request signal composed of the cosine signal plus components of uniform noise; b) the received signal superimposed on the filter bank; c) signals received after passing through the low-pass and high-pass filters. Note that the filters used here present gain in some passbands.

\section{Signal Detection}

Although the original signal may be correctly transmitted, received, and subdivided into a pair of filtered signals, the tag identification number, which the signal represents, is not detected yet. In this context, it becomes necessary to apply a logical decision-making process that extracts information about the possible carriers in these filtered signals and encodes the presence or absence in a binary identification sequence. This identification process comprises three procedures. The first procedure consists of applying the Fast Fourier Transform (FFT), which transforms it to a discrete frequency domain. The second procedure calculates the average powers of the filtered signals and noise. In particular, since these are discrete signals $x[n], 1 \leq n \leq N$, where $N$ is the number of samples for each signal, then Equation (3) applies. The Equation (3) takes the limit of the sum of the instantaneous energies of each sample.

$$
P_{x[n]}=\lim _{D \rightarrow+\infty} \frac{1}{2 D+1} \sum_{n=-D}^{D}|[x(t)]|^{2}
$$

Due to the noise's non-deterministic characteristic, it would be quite inaccurate to calculate the average noise power based only on a single example of a signal composed of only random noise 
components. Thus, we average the powers over 1,000 independent rounds of noise signals. We generate all these signals under the same initial conditions: the same number of samples and limits. Furthermore, the decision-maker compares the noise and the signals filtered by the low-pass and high-pass filters. Based on the comparison, the decision-maker performs the binary encoding of the identification number according to the rules:

- If the signal strength filtered by the low-pass filter is greater than the noise power, the first bit is 1. Otherwise, it is 0 .

- If the signal strength filtered by the high-pass filter is greater than the noise power, the second bit is 1 . Otherwise, it is 0 .

According to these rules, considering Figure 4, it is logical that the binary code generated by the proposed decision-maker for the toy example in the figure would be 11 , since there are carriers in both low and high frequency. It is worth noting that the filter bank is scalable for a higher number of orthogonal frequencies, increasing the addressable number of tags.

\section{EMULATION ASSESSMENT}

The randomness of noise hinders the decision-maker full functioning; it is essential to carry out an empirical study that estimated the decision-maker operating ranges. We use three different correction factors in this context, which reproduced varied and preferably low SNRs, 150 examples of request signals containing noise components were generated, 50 of them for each $F_{c o r r}$. Once created, each example of the set of signals is submitted to the same steps as described in Section IV-B. The results of the study are expressed in Figures 5(a) and 5(b).

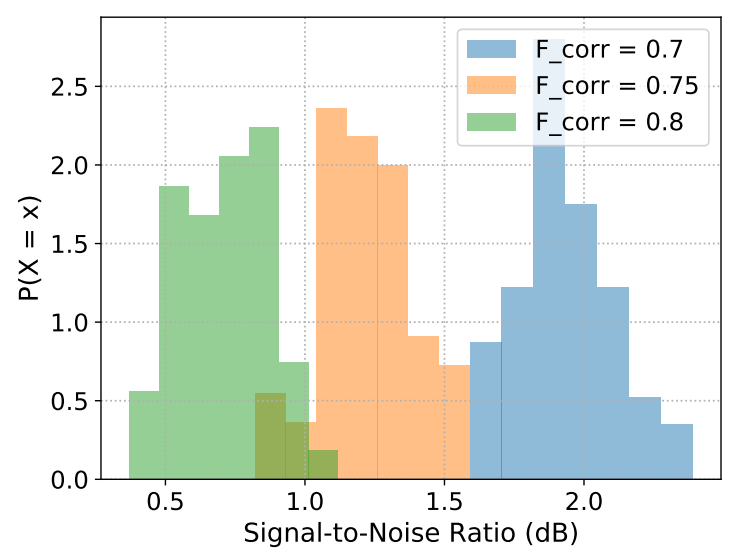

(a)

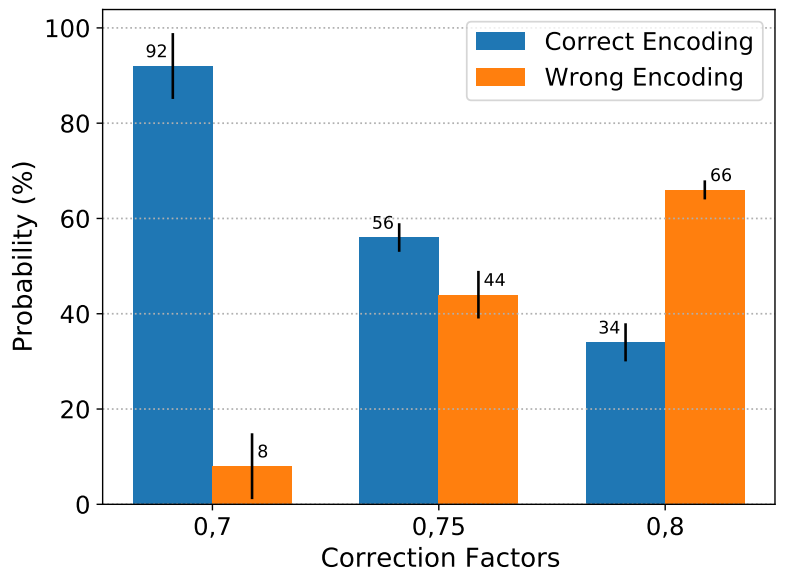

(b)

Fig. 5. Performance of the decision algorithm for the three correction values tested $\left(F_{\text {corr }}\right)$. It stands out the assertiveness above $90 \%$ even in noisy scenarios for an SNR approximately equal to $2 \mathrm{~dB}$. (a) SNR assessed values histogram, (b) Probability of a correct decision.

Figures 5(a) and 5(b) clearly show disparities between the decision-maker's performance when subjected to different SNRs. Low assertiveness rates, $34 \%$ and $56 \%$, of the decision-maker for $F_{\text {corr }}$ equal to 0.8 and 0.75 , respectively, are mainly due to the low SNR in these emulations, which sometimes leads to a noise power greater than the signal power. In these cases, the coding logic is impaired, interpreting any carriers' presence in the filtered signal as non-existent and, consequently, 
translating into binary codes containing a zero sequence. It is also clear that the decision-maker, for $F_{\text {corr }}$ equal to 0.7, has an assertiveness greater than $90 \%$ even in scenarios with SNRs in the range of 1.6 to $2.3 \mathrm{~dB}$.

\section{ANTENNA PROTOTYPE FOR THE RFID READER}

We have designed and manufactured a microstrip receiver antenna [19] operating at $915 \mathrm{MHz}$, according to the RFID system's operating requirements. Firstly, we simulate 26 scenarios, considering different substrates, such as FR-4 (Flame Retardant 4), Roger 5080, and Roger 6010, with varying thickness values, considering portable antennas, both for monopole and dipole antennas. Although all tested substrates showed good bandwidth values, directivity, and gain, we chose the epoxy-based FR4 substrate because it presents the lowest cost. Therefore, two PCB (Printed Circuit Board) dipole antennas are manufactured on the FR-4 substrate using the LPKF S103 prototyping machine, available at Laboratório de Antenas e Propagação (LAProp) at the Universidade Federal Fluminense (UFF), as depicted in Figure 6(a). Figure 6(b) shows the setup assembled for the measuring campaign. Figure 6(c) shows the manufactured antenna dimensions and reveals that the antenna is small and portable.

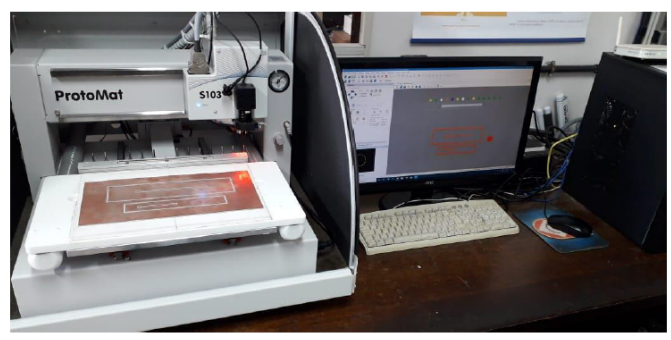

(a)

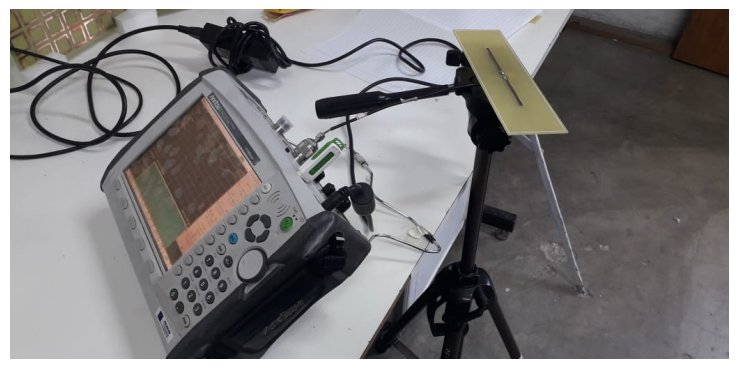

(b)

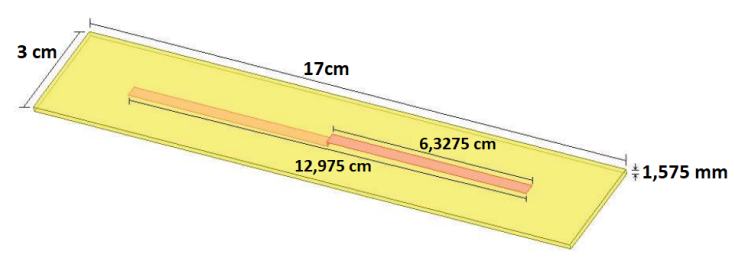

(c)

Fig. 6. (a) Antenna manufacturing process in LPKF S103 machine, (b) Experimental measurement of the reflection coefficient, and dimensions, (c) Dimensions of the proposed dipole antenna.

We perform experimental measurements of the antennas' reflection coefficient $\left(S_{11}\right)$ to evaluate our low-cost antenna prototype. Figures 7(a) and 7(b) compare the results of the simulated and the measured coefficient values. The antenna simulation reaches a reflection coefficient of $-22.79 \mathrm{~dB}$ at approximately $915 \mathrm{MHz}$. We obtain the experimental values for the signal reception of the manufactured antenna connecting the antenna to an Anritsu MS2034A vector network analyzer, configured as a spectrum analyzer, as shown in Figure 6(b). The transmitted signal was generated by an MG3700A signal generator and transmitted with one of the constructed antennas. The measured reflection coefficient in the antenna prototype reaches $-19.22 \mathrm{~dB}$ at an operating frequency close to $915 \mathrm{MHz}$. It is worth highlighting that the simulated and the measured reflection coefficient are compatible, evidencing the prototype's effectiveness. An alternative antenna based on a meander microstrip design is proposed by our research group in [18]. 


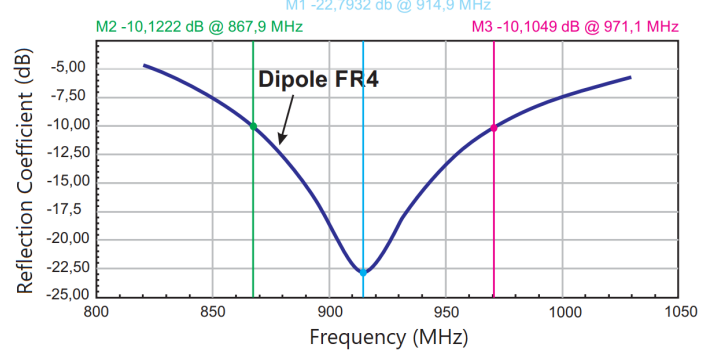

(a)

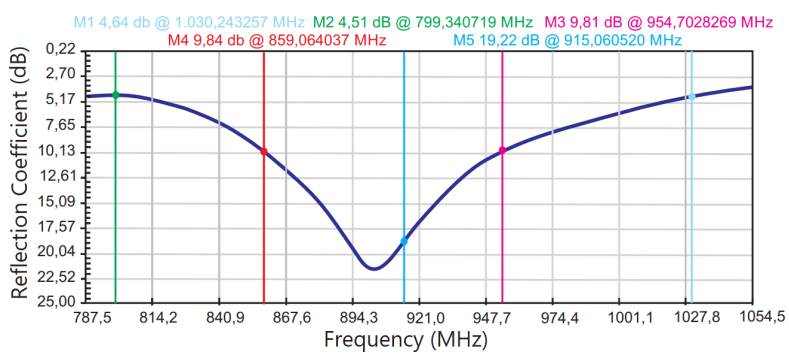

(b)

Fig. 7. Comparison of the results of the parameter $S_{11}$ in the simulated and measured scenarios. (a) Reflection coefficient simulated for the designed dipole antenna, (b) Reflection coefficient measured for the proposed dipole antenna.

\section{RELATED WORK AND DISCUSSION}

Ibrahim et al. describe a millimeter-sized identification chip operating in the range of 0.1 to 10 terahertz, with relatively low-energy levels supplied by photovoltaic diodes [13]. Applying the beam guidance function to the antenna array ensures more efficient communication, increasing the signal strength and range and reducing interference.

Nasimuddin et al. feature the microstrip antenna design for RFID reader with multiple bands, being able to work with a circularly polarized transmission system [15]. At the same time, Buffi et al. involve an array of 64 antenna elements, operating on a $2.4 \mathrm{GHz}$ operating range [16].

Regarding chipless RFID readers, Forouzandeh and Karmakar investigated the sources of interference in the RFID system, severely deteriorating the receiver sensitivity in detecting the low-power backscattered signal [27]. In this sense, the authors proposed implementing a self-interference compensation circuit capable of significantly reducing the substantial self-jamming signal leakage from the transmitter to the receiver before the receiver's saturation in the front-end. The circuit proved useful over singleantenna, dual-polarized antennas, and two-antenna readers for a frequency range of 4.3-7.3 GHz.

Other works concentrate on designing, simulating, and fabricating a low-cost UHF RFID reader antenna for surveillance and tracking high-value items in hospital environments [28]. The proposed planar microstrip antenna is operable at $915 \mathrm{MHz}$, and it is composed of recycled aluminum sheets consisting of two parallels plates with an L-shaped metal strip between them and four short plates for tuning. Although the antenna has managed to identify several items with UHF tags attached at various distances, the results also revealed a reflection coefficient of $-26.8 \mathrm{~dB}$ at the resonance frequency.

Garbati et al. [29] proposed a technique for chipless RFID reading based on a cross-polarization approach that relieves the need for tag-reader alignment. The proposed technique uses the Short-Time Fourier Transform (STFT), and an approach based on polarization diversity to retrieve the tag ID. Hence, it is possible to remove tag structural mode contribution and perform suitable excitation of the tag resonant scatterers to maximize the receiving tag energy. Nevertheless, experimental results in the laboratory indicated some limitations of the technique, including the mandatory use of two doubly polarized antennas and circulators, the increase in the reader's cost, and the reduction of the tag reading range depending on the antenna directivity.

In a multi-tag scenario, Deng et al. proposed a tag signal recovery method based on the widely linear minimum-mean-square-error (WLMMSE) criterion [30]. The anti-collision method considers the improper second-order statistics of backscattered tag signals, which cannot be easily achieved by a conventional linear model. Simulations in a MATLAB environment and real-world experiments confirm 
the method's efficiency in separating multiple overlapping tag signals within a single time slot and its adaptability into multi-antenna RFID systems to improve their throughput.

Boaventura et al. designed from scratch a UHF RFID reader capable of generating efficient powering waveforms [31], multisine (MS) signals. The proposal also included a preliminary evaluation of MS demodulation schemes using the developed hardware in conjunction with MATLAB. After measuring commercial passive RFID transponders' sensitivity performance when interrogated by powering waveforms, results show an enhancement of passive-backscattered radio communication range and the wireless power transfer capability.

Seeking to reduce RFID reader' costs, some works explore electronic printing, which employs high conductive inks in antenna manufacturing. Among these works, Xiao et al. proposed circularly polarized patch antennas for UHF RFID reader applications printed on an FR-4 substrate using the screen printing techniques [32]. Printed antennas proved to match available copper-based antennas in almost all the critical specifications, even though the conductive ink inherently renders lower conductivity for the printed conductors than the pure metal. A comparison between a commercial patch antenna and the proposed one reveals that the screen-printed antenna covers the desired frequency operating range more uniformly and successfully detected the tagged items arranged in the worst electromagnetic interference scenarios. The use of a circularly polarized antenna also allows the use of tags independently of its position and orientation.

Unlike previous works, in this paper, we proposed the design of the receiver system that considers both the execution of a Python application on standard and low-cost hardware and the prototyping of a small dipole antenna that operates at $915 \mathrm{MHz}$ with low reflection coefficient. Even manufactured with low-cost components, the proposed RFID system improves the performance of a professional RFID reader. The small dimensions and low weight of the developed antenna enhance its portability and allow it to be used in gates with any shape. The small dimensions of the Raspberry Pi circuit also makes it feasible to embed the receiver circuit in gates. This article presents the receiver part of the RFID, whereas article [17] presents most of the transmitting components. As presented in [17], experimental tests show that the tags present a good backscatter behavior when they are placed $25 \mathrm{~cm}$ apart from the reader antenna. Then the concept of the proposed RFID setup is considered feasible.

\section{CONCLUSION}

The object identification using radio frequency (RFID) is a technology that allows intelligent applications in the Internet-of-Things scenario. The development of modular receiver systems based on standard hardware is essential for the popularization of intelligent applications. Thus, in this paper, we proposed an RFID receiver system based on the Raspberry Pi microprocessor, with software developed in Python. The proposal emulation showed that, when receiving data scattered by a passive RFID tag, the proposal could separate and encode signals sent by the tag, with the decision-maker assertiveness close to $92 \%$ for SNR values $1.6 \mathrm{~dB}$ ranging up to $2.39 \mathrm{~dB}$. Additionally, it is worth noting that the software developed for the transmitter and the receiver is scalable and easily adapted to other types, quantities, and carriers' frequencies. We also designed and manufactured a low-cost dipole antenna prototype printed as a microstrip with a real reflection coefficient measured at $-19.22 \mathrm{~dB}$ at an operating frequency close to $915 \mathrm{MHz}$. We intend to carry out a complete system test with several tags and multiple users as future work.

Brazilian Microwave and Optoelectronics Society-SBMO Brazilian Society of Electromagnetism-SBMag received 31 Jan 2021; for review 31 Jan 2021; accepted 21 June 2021 (c) 2021 SBMO/SBMag (cc) BY

ISSN 2179-1074 


\section{ACKNOWLEDGMENT}

The authors would like to thank FAPERJ (E-26/210.524/2019 248292), FAPESP (2018/23062-5), CAPES (Code 0001), CNPq, RNP , and the City Hall of Niterói City for the funding that made this research possible.

\section{REFERENCES}

[1] J. Landt, "The history of rfid," IEEE Potentials, vol. 24, pp. 8-11, 2005.

[2] M. S. Sabarish, S. Srihari, T. S. Arunaa, and D. G. Kurup, "Simulation and design of a chipless passive rfid tag," in International Conference on Advances in Electronics Computers and Communications, pp. 1-3, 2014.

[3] C. M. Roberts, "Radio-frequency identification (rfid)," Elsevier Computer and Security, vol. 25, pp. 18-26, Oct 2007.

[4] ANATEL, "Resolution 680, june 27th 2017," accessed in July 2020. [Online]. Available: https://www.anatel.gov.br/ legislacao/resolucoes/2017/936-resolucao-680

[5] D. M. F. Mattos, P. B. Velloso, and O. C. M. B. Duarte, "An agile and effective network function virtualization infrastructure for the internet of things," Journal of Internet Services and Applications, vol. 10, no. 1, p. 6, 2019.

[6] L. H. A. Reis, L. C. S. Magalhães, D. S. V. de Medeiros, and D. M. F. Mattos, "An unsupervised approach to infer quality of service for large-scale wireless networking," Journal of Network and Systems Management, Apr. 2020.

[7] C. Tsirmpas, A. Rompas, O. Fokou, and D. Koutsouris, "An indoor navigation system for visually impaired and elderly people based on radio frequency identification (rfid)," Information Sciences, vol. 320, pp. 288-305, 2015.

[8] P. Sen, S. N. R. Kantareddy, R. Bhattacharyya, S. E. Sarma, and J. E. Siegel, "Low-cost diaper wetness detection using hydrogel-based rfid tags," IEEE Sensors Journal, 2019.

[9] A. S. Voulodimos, C. Z. Patrikakis, A. B. Sideridis, V. A. Ntafis, and E. M. Xylouri, "A complete farm management system based on animal identification using rfid technology," Computers and electronics in agriculture, vol. 70, no. 2, pp. 380-388, 2010.

[10] D. K. Klair, K.-W. Chin, and R. Raad, "A survey and tutorial of rfid anti-collision protocols," IEEE Communications Surveys \& Tutorials, vol. 12, no. 3, pp. 400-421, 2010.

[11] R. B. Di Renna, C. R. Corrêa, V. P. Magri, T. N. Ferreira, M. W. Silva, L. J. Matos, and J. A. Souza, "Novel design of a compact rfid chipless tag at 860, $915 \mathrm{mhz}$, and $2.4 \mathrm{ghz}$ bandwidth," Microwave and Optical Technology Letters, vol. 59, no. 10, pp. 2474-2479, 2017.

[12] N. Oliveira, L. Boaventura, T. Ferreira, V. Magri, J. Pereira, and D. Mattos, "Um sistema transceptor de baixo custo e alta confiabilidade para a leitura de etiquetas rfid passivas," in Anais do MOMAG 2020, Nov. 2020.

[13] M. Ibrahim, M. I. Khan, C. Juvekar, W. Jung, R. Yazicigil, A. Chandrakasan, and R. Han, “Thzid: A 1.6mm2 packageless cryptographic identification tag with backscattering and beam-steering at 260 ghz," 2020 Intenational Solid-State Circuits Conference (ISSCC), 2020.

[14] A. G. Araujo, J. S. Pereira, V. P. Magri, T. N. Ferreira, and P. V. Castellanos, "Desenvolvimento de um transmissor rfid utilizando arduino," in XXXVII Simpósio Brasileiro de Telecomunicações (SBrT2019), pp. 1-2, 2019.

[15] N. Nasimuddin, Z. N. Chen, and X. Qing, "Asymmetric-circular shaped slotted microstrip antennas for circular polarization and rfid applications," IEEE Transactions on Antennas and Propagation, vol. 58, no. 12, pp. 3821-3828, Dec. 2010.

[16] A. Buffi et al., "A focused planar microstrip array for $2.4 \mathrm{ghz}$ rfid readers," IEEE Transactions on Antennas and Propagation, vol. 58, pp. 1536-1544, May 2010.

[17] V. L. G. Mota, L. P. Boaventura, V. P. R. Magri, T. N. Ferreira, L. J. Matos, and V. N. H. Silva, "Simulation and fabrication of a low-cost rfid reader," in IEEE MTT-S Latin America Microwave Conference, May 2021.

[18] R. B. di Renna, R. D. R. Brasil, V. P. R. Magri, T. N. Ferreira, and L. J. Matos, "Design and simulation of broadband uhf microstrip meander antennas for an rfid reader," in IEEE MTT-S Latin America Microwave Conference, vol. 1, pp. 1-3, May 2021.

[19] D. Pozar, Microwave Engineering, 2nd ed. New York: John Wiley and Sons, 1998.

[20] I. Zalbide, E. D’Entremont, A. Jiménez, H. Solar, A. Beriain, and R. Berenguer, "Battery-free wireless sensors for industrial applications based on uhf rfid technology," in SENSORS, 2014 IEEE, pp. 1499-1502, 2014.

[21] A. A. Mbacke, N. Mitton, and H. Rivano, "A survey of rfid readers anticollision protocols," IEEE Journal of Radio Frequency Identification, vol. 2, no. 1, pp. 38-48, 2018.

[22] G. Maselli, M. Pietrogiacomi, M. Piva, and J. A. Stankovic, "Battery-free smart objects based on rfid backscattering," IEEE Internet of Things Magazine, vol. 2, no. 3, pp. 32-36, 2019. 
[23] J. Su, Z. Sheng, V. C. Leung, and Y. Chen, "Energy efficient tag identification algorithms for rfid: survey, motivation and new design,” IEEE Wireless Communications, vol. 26, no. 3, pp. 118-124, 2019.

[24] P. P. Vaidyanathan, Multirate Systems and Filter Banks. Prentice Hall, 1993.

[25] SCIPY, "Scientific computing tools for python," accessed in April 2021. [Online]. Available: https://www.scipy.org/

[26] G. Ozdemir and N. Karaboga, "A review on the cosine modulated filter bank studies using meta-heuristic optimization algorithms," Artificial Intelligence Review, vol. 52, no. 3, pp. 1629-1653, 2019.

[27] M. Forouzandeh and N. Karmakar, "Self-interference cancelation in frequency-domain chipless rfid readers," IEEE Transactions on Microwave Theory and Techniques, vol. 67, no. 5, pp. 1994-2009, 2019.

[28] V. Oliveira, G. Fontgalland, R. Rodrigues, T. Silveira, C. Melo, and I. Fontgalland, "Design, simulation and fabrication of low cost uhf rfid reader antenna for hospital applications," in 2018 11th German Microwave Conference (GeMiC), pp. 36-39, 2018.

[29] M. Garbati, E. Perret, R. Siragusa, and C. Halopè, “Toward chipless rfid reading systems independent of tag orientation," IEEE Microwave and Wireless Components Letters, vol. 27, no. 12, pp. 1158-1160, 2017.

[30] W. Deng, Z. Li, Y. Xia, K. Wang, and W. Pei, "A widely linear mmse anti-collision method for multi-antenna rfid readers," IEEE Communications Letters, vol. 23, no. 4, pp. 644-647, 2019.

[31] A. J. S. Boaventura and N. B. Carvalho, "The design of a high-performance multisine rfid reader," IEEE Transactions on Microwave Theory and Techniques, vol. 65, no. 9, pp. 3389-3400, 2017.

[32] G. Xiao, P. Aflaki, S. Lang, Z. Zhang, Y. Tao, C. Py, P. Lu, C. Martin, and S. Change, "Printed uhf rfid reader antennas for potential retail applications," IEEE Journal of Radio Frequency Identification, vol. 2, no. 1, pp. 31-37, 2018. 